\title{
Antibiotic Resistance and Beta-Lactamase Encoding Gene (Blavim) in Pseudomonas aeruginosa Isolated from Clinical Samples from Tanta University Hospitals
}

\author{
Aya G. Abdrabo*, Wesam S. Mohamed, Ghada A. Ahmed and Amany M. Abo El-Enein \\ Department of Clinical Pathology, Faculty of Medicine, Tanta University, Egypt \\ *Corresponding author
}

\section{Keywords \\ Antibiotic \\ resistance, \\ Pseudomonas \\ aeruginosa, beta- \\ lactamase, \\ Conventional PCR, Bla-VIM \\ Article Info \\ Accepted: \\ 12 March 2021 \\ Available Online: \\ 10 April 2021}

\section{A B S T R A C T}

High mortality rates are often associated with antimicrobial resistance, early detection of these resistant strains and their phenotypic and genotypic confirmation is necessary to decide the suitable therapy. To screen beta-lactamase production and its genes such as bla-VIM in Ps. aeruginosa strains isolated from hospitalized patients in Tanta University Hospitals. The study was carried out on 160 Pseudomonas aeruginosa isolates. Samples had been cultured, identified and tested for sensitivity. A phenotypic test (Double disc synergy test) was applied on the collected MDR isolates for detection of MBL enzyme production. Conventional PCR was performed for the detection of the resistance gene(blaVIM). 100 isolates were multi-drug resistant according to (Modified Kirby Bauer disc diffusion method), with sensitivity to some drugs specially Amikacin (20\%). Phenotypic test for MBL resulted in (38\%) positive isolates and (62\%) were negative ones. Multiplex PCR detected gene (bla-VIM) in (30\%) isolates and it was absent in (70\%) of the isolates. High prevalence of MBL among multi-drug resistant $P$. aeruginosa is thought to be due to the uncontrolled consumption of antimicrobials. Rapid identification of such strains is needed to decrease mortalities.

\section{Introduction}

Pseudomonas aeruginosa is an important opportunistic pathogen in nosocomial infections and responsible for high mortality rates (Rostami et al., 2018). The presence of efflux pumps, reduced permeability of the outer membrane and secretion of the $\beta$ - lactamase enzyme are the main prominent features of this bacteria for the inherent resistance to many antibiotics (Rehman et al., 2018).

The most important carbapenemases produced by $P$. aeruginosa are zinc-dependent metallobeta-lactamases (mbls) capable of 
hydrolyzingimipenem, meropenem and cephalosporins (Poole, 2011). There are various MBL genes among carbapenemresistant $P$. Aeruginosa including Verona integron-encoded MBL (VIM), Germany imipenemase $(G I M)$, imipenemase $(I M P)$ and others (Hong et al., 2015).Each MBL gene is encoded by specific genetic elements including transposons, integrons, plasmids, or on the chromosome, carrying genes encoding determinants of resistance to several antibiotics.

Moreover, these genetic determinants are transferable to other Gram-negative species, extending the antimicrobial resistance rate and complicating the treatment of infected patients (Hong et al., 2015).

PCR is considered a cost-saving application that can be used for locating specific sets of genes (blavim, blaspmand bla IMP) with the aid of oligonucleotide primers (Besli et al., 2018). The treatment and control of $P$. aeruginosa infections acquired both in the community and in hospitals is a serious therapeutic challenge, for which the selection of appropriate antibiotics for initiation of therapy is essential.

The spread of this bacterium is difficult to control with disinfectants and antibiotics due to the many intrinsic and acquired mechanisms of resistance (Breidenstein et al., 2011, Chatterjee et al., 2016). Therefore, early detection of these strains is important for clinicians in order to select appropriate antibiotics and to reduce mortalities (Lee and Ko, 2012).

\section{Materials and Methods}

The study was carried out in the Clinical Pathology Department, faculty of medicine, Tanta University, on 160 pseudomonas aeruginosa isolates during the period of research from August 2018 to August 2019from I.C.Us of Internal medicine, chest, emergency and anaesthesia departments, Tanta University Hospitals.

All samples were collected under complete aseptic precautions. Thesamples included: Sputum samples, Broncho-alveolar lavage (BAL), Septic wound swabs, Urine samples and Blood samples. All samples were cultured and then Gram stain smears were made from the colonies and examined microscopically.

Urine samples were cultured also on CLED agar by inoculating a loop $10 \mu 1$ of well-mixed uncentrifuged urine using a sterile calibrated bacteriological loop by surface streak method.

Antimicrobial susceptibility of the isolates was determined by modified Kirby Bauer disc diffusion method on Muller Hinton agar plates according to Clinical and Laboratory Standard Institute guideline 2019 (CLSI, 2019)

IMP-EDTA combined disc test was done for detection of MBL among imipenem resistant isolates.

Genotypic detection of mbls resistance genes was performed using a PCR technique using the following primer:

\section{$5^{`} \rightarrow 3^{`}$ GTTTGgTCGC ATATCG CAAC, $3^{\prime} \rightarrow 5{ }^{\prime}$ AATGCGCAGCACCAGGATAG.}

\section{Statistical Analysis}

A statistical analysis was conducted, using the mean and standard deviation. Data were fed to the computer and analyzed using IBM SPSS software package version 20.0 (Kirkpatrick \& Feeney, 2013).Chi-square test was used as a test of significance for qualitative data, Pvalue considered significant when $<0.05$. 


\section{Results and Discussion}

\section{Regarding the distribution of Pseudomonas growth among male and female patients}

Males (59.3\%) are much higher than females(40.7\%). Among all 160 Pseudomonas isolates, samples from pus $(35.6 \%)$ and urine $(25 \%)$ showed the largest percentages followed by blood (16.9\%) and sputum $(13.7 \%)$ then broncho-alveolar lavage showed the least percentage $(8.75 \%)$.

\section{Regarding the distribution of samples among departments}

The highest percentage of pus was found in Emergency I.C.U (42.1\%), while the highest percentage of blood samples was observed in Anesthesia I.C.U. (44.4\%). The highest number of urine samples was found in Emergency I.C.U (37.5\%). The highest percentage of sputum and broncho-alveolar lavage samples were found in Chest I.C.U $(63.6 \%, 78.5 \%)$ respectively. There was a statistically significant difference between the four departments regarding distribution of the source of samples (P-value <0.001) (Table 1).

Antibiotic resistance study through disc diffusion test

Antibiotic resistance study through disc diffusion test showed 100isolates with multidrug resistance (MDR) (62.5\%) with sensitivity to some antibiotics especially Amikacin (20\%)(Figure 1).

\section{Phenotypic study}

Phenotypic study was applied on the isolates to indicate the production Metallo $\beta$ lactamases, positive samples were (38\%) (38) out of (100), while the negative cases were (62\%) (62) samples.

\section{Genotypic detection by Conventional PCR}

Genotypic detection by Conventional PCR was conducted on all 100 resistant isolates. (bla VIM) MBL gene was detected in (30) isolates (30\%) and 70 isolates showed no gene(Figure 2).

Agarose gel electrophoresis of MPL gene amplified from 15 different isolates. Lane $\mathrm{M}$ is 100 bp DNA markers. Lanes 2 and 7 show no amplified product. The other lanes show amplified products of blavimgene.

\section{Calculating Sensitivity and Specificity values}

Calculating Sensitivity and Specificity values for phenotypic test using PCR test as a gold standard one: showed 100\% sensitivity and $88.75 \%$ specificity. So, phenotypic tests are considered valuable, practical and simple method for detection of resistant strains of $P$. aeruginosa. Beside Double disk synergy test PCR is considered the gold standard for the detection of MBL activity.

$P$. aeruginosa is a major nosocomial pathogen worldwide; infections are difficult to treat due to the intrinsic resistance of the strains, and its remarkable capability of acquiring additional resistance mechanisms to different groups of antimicrobials (Saber et al., 2015). Carbapenem antibiotics used as a treatment of resistant infections are extremely threatened cause of the emergence carbapenemases (Diene \& Rolain, 2014). The capability of (MBL) to hydrolyze almost all $\beta$-lactams, and their combination with mobile genetic elements as plasmids and transposons, induce their rapid spread (Diene \& Rolain, 2014).

Regarding the importance of $P$. aeruginosa infections and the evolving multidrug resistance strains (MDR), the phenotypic and genotypic identification is required (Amini \& Ebrahimzadeh Namvar, 2019). 
The aim of this study was to screen betalactamase production and its genes such as bla-VIM in P. aeruginosa strains isolated from hospitalized patients. In the present study the percentage of males was $(59.3 \%)$ while females was $(40.7 \%)$ of the isolates, unlike Anil \& Shahid, 2013who showed female predominance $(55 \%)$.

Also, Amini \& Ebrahimzadeh Namvar, 2019 reported similar results to the present study showing a predominance of males more than females $(62 \%, 38 \%$ respectively).The different results of sex distribution may be due to different epidemiological regions.

The (160) isolates are categorized in this study according to their origin into $(35.6 \%)$ of isolates from pus, $(25 \%)$ isolates from urine, (16.9\%) of isolates from blood, (13.7\%) of isolates from sputum and $(8.7 \%)$ of isolates from broncho-alveolar lavage.

This was similar to Hashem et al., 2017 and Anil \& Shahid, 2013 who reported highest number of samples from pus (43\% \& 27\%) respectively. Adversely, Chaudhary \& Payasi, 2013 reported that the highest number of isolates originated from blood (46.7\%).Distribution of samples origin may vary due to different environments among hospitals and health care settings.

In the present study, pus and urine samples were found to be the most prevalent among Emergency I.C.U samples (42.1\%, 37.5\%) respectively. Sputum samples were most prevalent in Chest I.C.U with percent of $(63.6 \%)$ of total sputum samples. Similar findings are recorded in bronchoalveolar lavage samples, as most of them were collected from Chest I.C.U (78.5\%). There was a statistically significant difference between the four departments regarding the source of samples ( $\mathrm{P}$ value $<0.001$ ). On the other hand, Hussain et al., 2017 found out that the highest percentage of pus was from burn unit in accordance with Yousefi et al.,2010 who reported percentage of $(46.15 \%)$ in pus samples isolated from burn units.

These findings may have vital role in the establishment of hospital infection control policy. The difference in the results could be due to different inclusion criteria and number of isolates.

In this study, when the discs of antibiotics were applied on the collected isolates there were 100 multi-drug resistant samples $(62.5 \%)$ according to (Modified Kirby Bauer disc diffusion method) and showed sensitivity to some antibiotics especially Amikacin (20\%), Ciprofloxacin (5\%) and Imipenem (3\%).

These results are nearly similar to Amini \& Namvar, 2019 who reported (61.9\%) MDR isolates and Ellappan et al., 2018 and Mohamed \& Raafat, 2011 who reported (92.8\% \& 95\%) MDR respectively. Adversely, Chaudhary \& Payasi 2013 reported 100\% resistance against imipenem.

In the present study, the most sensitive antibiotics were Amikacin(20\%), Ciprofloxacin (5\%) and Imipenem (3\%). Kanungo et al., 2006 reported (20.7\%) sensitivity to Amikacin.

However, Mansoor et al., 2009 showed higher sensitivity rates with (96\%) to Amikacin, $(85 \%)$ to Ciprofloxacin and (76\%) to Imipenem. Thus, monitoring of resistant $P$. aeruginosa isolates and the identification of the factors associated with this resistance is essential in order to prevent the emergence of pan-drug resistance among clinical isolates of P. aeruginosa (Dantas et al., 2014). 
Table.1 Prevalence of sample sources among departments.

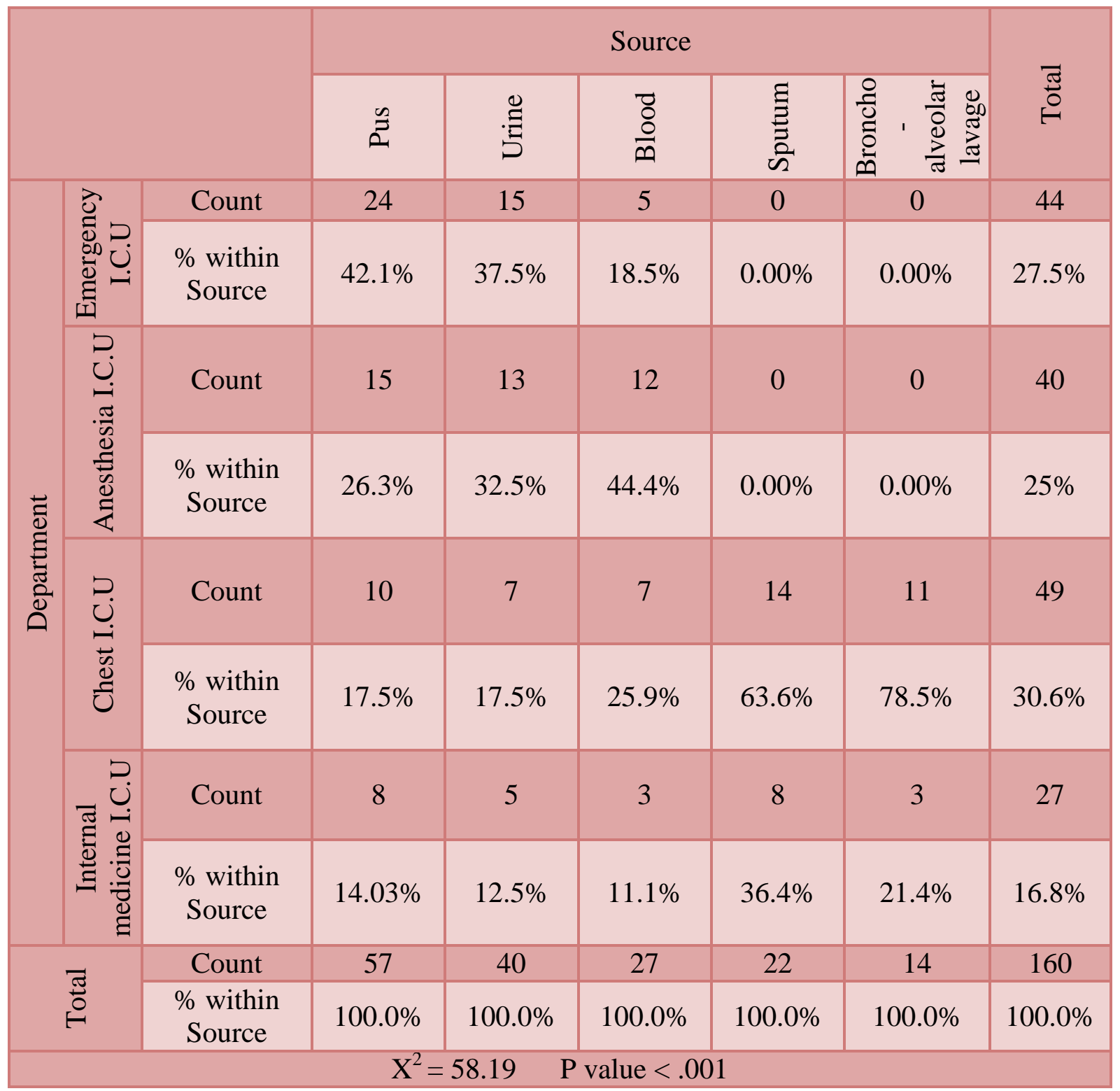


Fig.1 Plate of antibiotic sensitivity for a case of MDR Pseudomonas aeruginosa

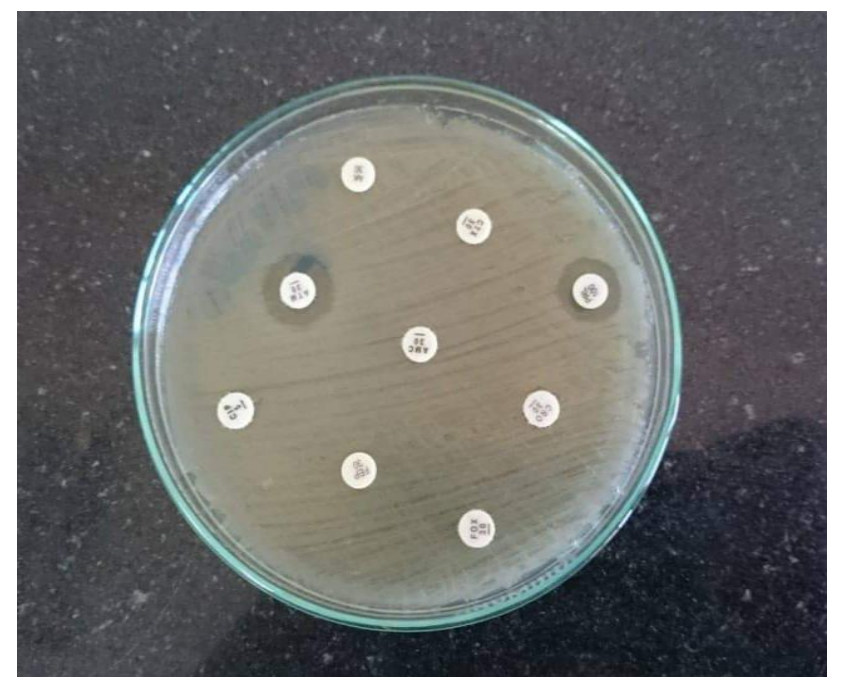

Fig.2 Detection of (blavim) gene by Multiplex PCR.

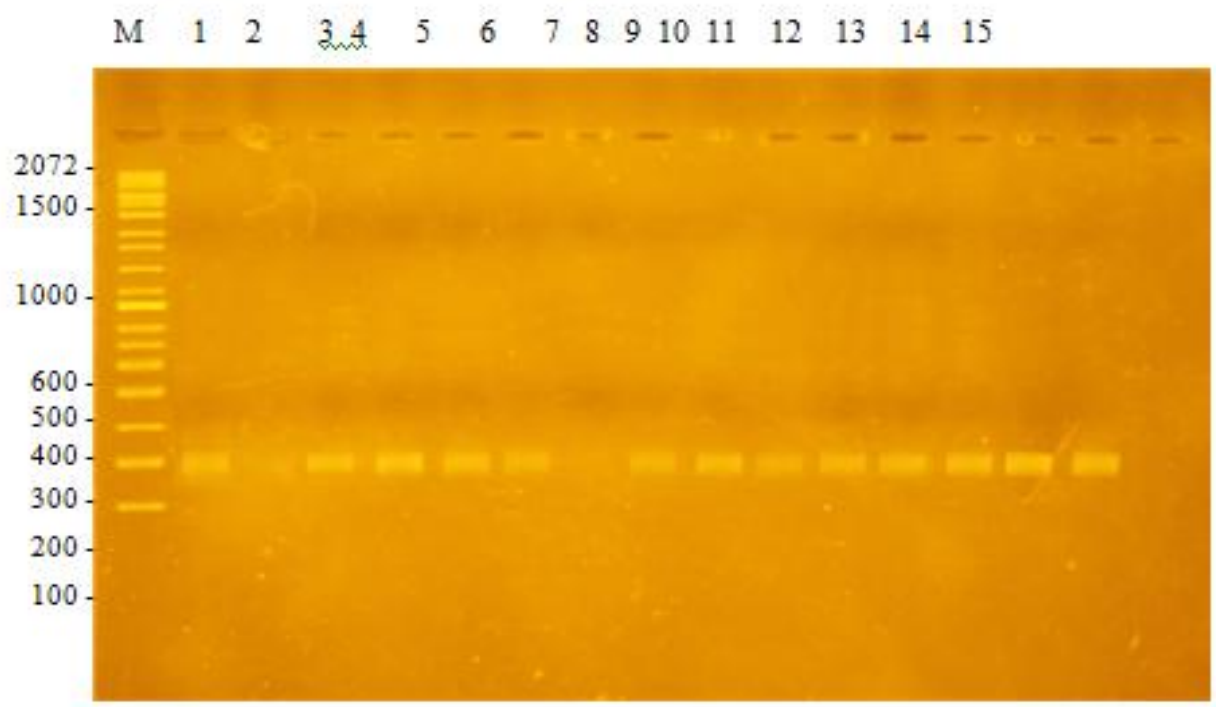

The phenotypic test (Double disc synergy test) was applied on the collected MDR isolates for detection of MBL enzyme production in these samples resulting in $(38 \%)$ positive isolates and $(62 \%)$ negative. Nearly similar results were shown in Zubair \& Iregbu 2018 study which reported that phenotypic test was positive in $(11 \%)$ of resistant isolates. In this study, blavimgene was detected in $(30 \%)$ of isolates of total100 MDR samples. All the 30 isolates were phenotypic positive(Metallo $\beta$ - lactamase producers). While the 8 phenotypic positive isolates were lacking blavimgene suggesting the presence of other MBL genes as GIM or SPM for example.

Though the good performance of phenotypic screening test for MBL, they are not specific as false positive results were reported in our study. Amudhan et al., 2012 has agreed with our results reporting that among (61) $P$. Aeruginosa isolates (bla VIM) was detected in 
(34) isolates (55.7\%). However, Zubair \& Iregbu 2018 has reported detection of blaVIM gene in only (5) isolates of (22) MBL producers $(22.7 \%)$.

Despite of several phenotypic tests, PCR is considered the gold standard method which is unfortunately not available and not used for routine diagnostic laboratories (Smith et al., 2016).

In the present study, phenotypic detection of MBL showed 100\%sensitivity and 88.75\%.This was like Ellapan et al., 2018 who recorded $(66.7 \%)$ sensitivity and $(95 \%)$ specificity. Although genotypic characterization is considered the gold standard, but molecular methods is complex, requires special training, and is associated with higher costs (Ashok et al., 2016).

As Beta-lactamases are globally distributed, and their incidence is variable, awareness of their resistance rate is essential in the prevention of their spread and selection of appropriate treatment options.

MBL producing Pseudomonas aeruginosa is extremely resistant to the $\beta$ lactam group of drugs and Cephalosporins.

Amikacin, Ciprofloxacin and Imipenem were the most sensitive antibiotics and hence they should be kept as reserved drugs.

Although the gold standard for the detection of Metallo $\beta$-lactamase activity was genotypic tests, but the phenotypic tests can be used as a screening test.

\section{References}

Amini, A., Ebrahimzadeh, Namvar, A., (2019). Antimicrobial Resistance Pattern and Presence of BetaLactamase Genes in Pseudomonas aeruginosa Strains Isolated from Hospitalized Patients, Babol-Iran. JMed Bacteriol. $8(1,2)$ : pp.4

Amudhan, M. S., Sekar, U., Kamalanathan, A. And Balaraman, S. (2012). Blaimp and blavimmediated carbapenem resistance in Pseudomonas and Acinetobacter species in India. The Journal of Infection in Developing Countries, 6(11), 757-762

Anil, C., and Shahid, R. M. (2013). Antimicrobial susceptibility patterns of Pseudomonas aeruginosa clinical isolates at a tertiary care hospital in Kathmandu, Nepal. Asian J Pharm Clin Res, 6(3), 235-85-50.

Ashok A K, Jaryal S C, Thakur K, Sood A, Gupta P K, et al., (2016). Detection of Inducible and Non-inducible (constitutive) ampc $\beta$-lactamase producing Gram-Negative Bacteria among Family Enterobacteriaceae by Two Phenotypic Methods-Disk Antagonism Test (DAT) and ampc disk Test at a Tertiary Care Hospital, Himachal Pradesh, India. International Journal of Current Microbiology and Applied Sciences;5(4):133-139

Besli, Y., Bayramoglu, G., Tosun, I., Kaklikkaya, N., \& Aydin, F. (2018). Molecular Epidemiology and Clinical Characteristics of Metallobetalactamase Producing Pseudomonas aeruginosa Isolates/Metallo-BetaLaktamaz Ureten Pseudomonas aeruginosa Suslarinin Molekuler Epidemiyolojisive KlinikOzellikleri. Mediterranean Journal of Infection, Microbes and Antimicrobials, 7, 1H-1H.

Breidenstein, E. B. M., de la Fuente-Nu' ñez, C. and Hancock, R. E. W. (2011). Pseudomonas aeruginosa: all roads lead to resistance. Trends Microbiol 19, 419-426.

Chatterjee, M., Anju, C. P., Biswas, L., Anil 
Kumar, V., Gopi Mohan, C., Biswas, R., (2016). Antibiotic resistance in Pseudomonas aeruginosa and alternative therapeutic options Int $\mathrm{J}$ Med Microbiol 306, 48-58.

Chaudhary, M., \& Payasi, A. (2013). Rising antimicrobial resistance of Pseudomonas aeruginosa isolated from clinical specimens in India. JProteomics Bioinform, 6(1), 5-9.

Clinical and Laboratory Standards Institute CLSI. (2019). M100ED29:2019Performance Standards for Antimicrobial Susceptibility Testing, $29^{\text {th }}$ Edition. CLSI, Wayne, P A

Dantas, R. C., Ferreira, M. L., Gontijo-Filho, P. P., \& Ribas, R. M. (2014). Pseudomonas aeruginosa bacteraemia: independent risk factors formortality and impact of resistance on outcome. Journal of medical microbiology, 63(12), 1679-1687.

Diene, S. M., \& Rolain, J. M. (2014). Carbapenemase genes and genetic platforms in Gram-negative bacilli: Enterobacteriaceae, Pseudomonas and Acinetobacter species. Clinical Microbiology and Infection, 20(9),831-838.

Ellappan, K., Narasimha, H. B. and Kumar, S., (2018). Coexistence of multidrug resistance mechanisms and virulence genes in carbapenem resistant Pseudomonas aeruginosa strains from a tertiary care hospitalin South India. Journal of global antimicrobial resistance, 12, pp.37-43.

Hashem, H., Hanora, A., Abdalla, S., Shaeky, A., Saad, A., (2017). Dissemination of metallo-b-lactamase in Pseudomonas aeruginosa isolates, in Egypt: mutation in blavim-4. APMIS; 125: 499-505.

Hong, D. J., Bae, I. K., Jang, I-H., Jeong, S. H., Kang, H-K., Lee, K., (2015). Epidemiology and characteristics of metallo- $\beta$-lactamase-producing

Pseudomonas aeruginosa. Infection \& chemotherapy. 47(2):81-97

Hussain, M. S., Nasir, B., Shahid, H., Sarwar, F., \& Ejaz, A. (2017).Prevalence and antibiogram of Pseudomonas aeruginosa isolated from clinical samples at a tertiary care hospital. Blood, 2, 9

Kanungo, R., Srinivasan, S., \& Devi, S. (2006). Emerging resistance to carbapenems in hospital acquired Pseudomonas infection: A cause for concern. Indian Journal of Pharmacology, 38(4), 287

Kirkpatrick, L. A., and Feeney, B. C., (2013). A simple guide to IBM SPSS statistics for version 20.0. Student ed. Belmont, Calif. Wadsworth, Cengage Learning. 2013

Lee, J. Y., Ko, K. S., (2012). Oprdmutations and inactivation, expression of efflux pumps and ampc, and metallo-blactamases in carbapenem resistant Pseudomonas aeruginosa isolates from South Korea. Int JAntimicrob Agents; 40:168-72.

Mansoor, T., Musani, M. A., Khalid, G., \& Kamal, M. (2009). Pseudomonas aeruginosa in chronic suppurative otitis media: Sensitivity spectrum against various antibiotics in Karachi. $\mathbf{J}$ Ayub Med Coll Abbottabad, 21(2), 120-3.

Mohamed, N. M., \& Raafat, D. (2011). Phenotypic and Genotypic Detection of Metallo-beta-lactainases in Imipenemresistant Acinetobacter baumannii Isolated from a Tertiary Hospital in Alexandria, Egypt. ResJ Microbiol, 6, $750-760$

Poole, K., (2011). Pseudomonas aeruginosa: resistance to the max. Front Microbiol. 2(65)

Rehman, A., Patrick, W. M., \& Lamont, I. L. (2018). Mechanisms of ciprofloxacin 
resistance in Pseudomonas aeruginosa: new approaches to an old problem. Journal of medical microbiology, 68(1), 1-10

Rostami, S., Sheikh, A. F., Shoja, S., Farahani, A., Tabatabaiefar, M. A., Jolodar, A., \& Sheikhi, R. (2018). Investigating of four main carbapenem-resistance mechanisms in high-level carbapenem resistant Pseudomonas aeruginosa isolated from burn patients. Journal of the Chinese Medical Association, 81(2), 127-132.

Saber, Y., Atef, M., Ziad, A., Memish, Z. A., (2015). The molecular basis of blactamase production in Gramnegative bacteria from Saudi Arabia. JMed Microbiol;64:127-36

Smith, M., Diederen, B., Scharringa, J., Leversteijn-van Hall, M., Fluit, A. C., et al., (2016). Rapid and accurate detection of carbapenemase genes in Enterobacteriaceae with the Cepheid XpertCarba-R assay. Journal of Medical Microbiology; 65(9): 951953.

Yousefi, S., Farajnia, S., Nahaei, M. R., Akhi, M. T., Ghotaslou, R., Soroush, M. H., \& Jazani, N. H. (2010). Detection of metallo- $\beta$-lactamase- encoding genes among clinical isolates of Pseudomonas aeruginosa in north west of Iran. Diagnostic microbiology and infectious disease,68(3), 322-325.

Zubair, K. O., \& Iregbu, K. C. (2018). Resistance pattern and detection of metallo-beta-lactamase genes in clinical isolates of Pseudomonas aeruginosa in a central Nigeria Tertiary Hospital. Nigerian journal of clinical practice, 21(2), 176-182.

\section{How to cite this article:}

Aya G. Abdrabo, Wesam S. Mohamed, Ghada A. Ahmed and Amany M. Abo El-Enein. 2021. Antibiotic Resistance and Beta-Lactamase Encoding Gene (Blavim) in Pseudomonas aeruginosa Isolated from Clinical Samples from Tanta University Hospitals. Int.J.Curr.Microbiol.App.Sci. 10(04): 386-394. doi: https://doi.org/10.20546/ijcmas.2021.1004.041 